\title{
Behind the scenes - data processing and quality assurance for the ICSD
}

\section{Annett Steudel, Stephan Rühl}

FIZ Karlsruhe - Leibniz-Institut für Informationsinfrastruktur, Eggenstein-Leopoldshafen, Germany;

annett.steudel@fiz-karlsruhe.de

The Inorganic Crystal Structure Database (ICSD) has been collecting published crystal structures for more than 40 years. In addition, the database offers the structures in curated and extended form. In the process of adding a structure to the database, a series of tests are run to verify data integrity and correctness. Furthermore, the data is enriched with additional or missing information, which can help to detect possible discrepancies. Some of the procedures used will be explained here, and examples will be given to show how careful evaluation of crystallographic parameters and the addition of missing parameters improves the quality of the crystal structure entry.

Keywords: data curation; crystal structures; databases; inorganic chemistry; metalorganic chemistry 